\title{
Association of Preoperative mid-stream urine culture, Renal pelvic urine culture and Renal stone culture in the detection of Systemic inflammatory response syndrome/urosepsis post Percutaneous Nephrolithotomy
}

\author{
Neeraj Thapa $^{1^{*}} \mathbb{D}$, Suman Baral ${ }^{1}$, Nabina Maharjan², Raj Kumar Chhetri1 ${ }^{1}$, \\ Sumnima Acharya ${ }^{3}$ (D), Kamal Karki ${ }^{1}$, Ranjit Mahato ${ }^{1}$ \\ 'Department of Surgery, ${ }^{2}$ Department of Microbiology, ${ }^{3}$ Department of Radiology, Lumbini Medical College
}

\begin{abstract}
Introduction: Urosepsis post Percutaneous Nephrolithotomy (PCNL) is a dreaded complication with high mortality rate. Objective: To find the association of preoperative midstream urine culture (PMUC), renal pelvic urine culture (RPUC) and renal stone culture (RSC) in the post-operative development of Systemic Inflammatory Response Syndrome (SIRS) and urosepsis. Methods: It was a prospective cross-sectional observational study. The study included all symptomatic patients meeting the inclusion criteria who underwent Percutaneous Nephrolithotomy from $18^{\text {th }}$ August, 2019 to $28^{\text {th }}$ March, 2020. PMUC, RPUC and RSC were done and analyzed accordingly. Results: A total of 140 (73 males, 67 females) patients underwent PCNL. PMUC was positive in 15\% (21/140) as compared to RPUC and RSC which were $7.9 \%(11 / 140)$ and $4.3 \%(6 / 140)$ of total cases. None of the patients had simultaneous culture positivity in all the three types of specimens. Only two (1.42\%) patients had simultaneous positivity in pelvic urine culture and stone culture. The organisms obtained in pelvic urine culture and stone culture were same i.e. Klebsiella and Escherichia coli respectively. Only two (1.42\%) patients developed SIRS post PCNL, where in both the cases stone culture were positive but PMUC and RPUC were negative. Urosepsis was found in none of the patients. In the Fischer Exact test PMUC and RPUC were not statistically significant in the detection of SIRS post PCNL. Whereas only RSC showed statistical significance in the detection of SIRS. Conclusion: Stone culture has high prediction for SIRS and it might be considered for patients undergoing PCNL in order to prevent stone related infective complications.
\end{abstract}

Keywords: Percutaneous nephrolithotomy, Sepsis, Urine culture

\section{*Correspondence to:}

Dr. Neeraj Thapa, MS

Assistant Professor, Surgery Department, Lumbini Medical College,

Email: drneerajthapa@gmail.com,

Submitted: April 18, 2020

Accepted: May 25, 2020

To cite: Thapa N, Baral S, Maharjan N, Chhetri RK, Acharya S, Karki K, et al. Association of Preoperative mid-stream urine culture, Renal pelvic urine culture and Renal stone culture in the detection of Systemic inflammatory response syndrome/urosepsis post Percutaneous Nephrolithotomy. JGMC Nepal. 2020;13(1):14-18. DOI: 10.3126/igmcn.v13i1.29378

\section{INTRODUCTION}

Percutaneous Nephrolithotomy (PCNL) is considered as the procedure of choice for a complex and large volume nephrolithiasis in all age groups. ${ }^{1-3}$ But the procedure is not devoid of complications as fever occurs in $21 \%$ to $39.8 \%$ of patients undergoing PCNL. ${ }^{4}$ The incidence of urosepsis is $0.3 \%$ to $9.3 \%$ and the septic shock is $1 \%$ in which the mortality reaches up to 66 to $80 \%$ post PCNL. ${ }^{5-8}$ Hence, the factors leading to urosepsis in PCNL should be considered seriously in order to prevent the dreaded complications.

Factors responsible for post-operative urosepsis are the number of stones, duration of operation, bleeding and presence of residual stones. ${ }^{9}$ Despite these factors, preoperative Urinary Tract Infection (UTI) also leads to post-operative urosepsis if urine is not made sterile before PCNL. Antibiotic prophylaxis is advised by the American 
Urological Association (AUA) for the prevention of postoperative urosepsis but despite prophylaxis the incidence of urosepsis is $8-10 \% .^{10}$ Thus control of urinary tract infection (UTI) is needed which is best correlated by Renal Pelvic Urine Culture (RPUC) and Renal Stone Culture (RSC) as compared to Pre-operative Midstream Urine Culture (PMUC). ${ }^{11}$

It is imperative to control UTI to prevent post-operative urosepsis and reduce the morbidity and mortality. Hence, this study aimed to find the association of PMUC, RPUC and RSC in the post-operative development of Systemic Inflammatory Response Syndrome (SIRS) and urosepsis.

\section{MATERIALS AND METHODS}

This was a prospective cross-sectional observational study conducted at the Department of Surgery, Lumbini Medical College and Teaching Hospital effectively from $18^{\text {th }}$ August, 2019 to $28^{\text {th }}$ March, 2020. Ethical approval was taken from the Institutional Review Committee of Lumbini Medical College and Teaching Hospital (IRC-LMC 06-G/019).

The sample size was calculated using the formula: Sample Size $(S)=Z^{2} P q / d^{2}$, where: $Z=$ Confidence Level of $95 \%$ (Standard Value -1.96), P= Estimated Prevalence of Renal Stones (That corresponds to around $10 \%)^{12}, \mathrm{q}=1-\mathrm{P}, \mathrm{d}=$ Margin of error (0.05), Calculating sample size from above formula, the minimal sample required was 140 . So, we decided to take the number of samples of 145 .

All patients with renal stone size more than $10 \mathrm{~mm}$ in the largest dimension were included in the study, whose stone burden was calculated by multiplying the largest two dimensions of the stone. Patients not giving consent for the study, those having persistent preoperative midstream urine culture positivity and untreated coagulopathy were excluded from the study. Five cases were excluded from the study as three cases did not give the consent and the rest of the two had persistent coagulopathies. Routine preoperative and diagnostic investigations including ultrasonography of abdomen and pelvis, intravenous urogram and the preoperative midstream urine culture (PMUC) were done in all patients along with Computed Tomography -Intravenous urogram (CT-IVU) in selected cases. All patients were treated with empirical antibiotics for three days before the PMUC report arrived and the PMUC negative cases were taken for surgery, whereas for PMUC positive cases, culture specific antibiotics were administered for two weeks prior to the surgery and the PMUC was repeated. In every patient surgery was performed only when the PMUC report came out to be negative. All patients received intravenous antibiotics i.e. third generation cephalosporin (Cefoperazone Sulbactum combination) of dose $1.5 \mathrm{gms}$ for adults and $750 \mathrm{mg}$ for children along with aminoglycosides (Amikacin) of dose $15 \mathrm{mg} / \mathrm{kg} /$ day a day prior to the surgery according to the local hospital protocol which was carried out till the third postoperative (POD) day which was then subsequently converted into oral antibiotics continued up to seven days post-operatively.

All patients received the above mentioned intravenous antibiotics one hour before the procedure. Five French (Fr) ureteric catheter was introduced into ipsilateral ureter via 20 Fr cystoscope (Karl Storz, Germany) in lithotomy position and foley's catheterization was done. Later, the position was changed into prone and under fluoroscopic guidance using $\mathrm{C}$-arm (Allengers, India) after the instillation of urografin $76 \%$ via ureteric catheter, targeted calyceal puncture was done using 18 Gauge (G) needle. Pelvic urine was aspirated from the puncture site and was sent for the culture in $3 \mathrm{ml}$ sterile urine within one hour of collection. Subsequent dilatation of the track with the help of fascial dilators (Cook, Germany) was done using Seldinger's technique with the introduction of 0.035 inches guide wire. After the dilatation of track Amplatz sheath of size 20 Fr were used. Nephroscope (Wolf, Germany) of size $18 \mathrm{Fr}$ was introduced and the stones were visualized and broken down into fragments using lithotripter (Nidhi lith, India). The stones were taken out using forceps. The fragmented stones were washed with normal saline and sent in fresh normal saline for the culture. Double J stent (DJ stent) was routinely kept in all patents. Nephrostomy drain of size $18 \mathrm{Fr}$ was also placed routinely at the end of the procedure. Nephrostomy drains were removed on the third postoperative day while DJ stents were removed three weeks after the procedure. Patients were closely monitored for any signs of SIRS (Systemic Inflammatory Response Syndrome) and urosepsis postoperatively. UTI was defined as urine test having pus cells equivalent to or more than five in number per high power field in microscopic examination. ${ }^{13}$ SIRS was defined as patients having two or more of the following criteria i.e. hypothermia (body temperature less than 36 degree Celsius) or hyperthermia (body temperature more than 38 degree Celsius), leukocytosis (total leucocyte count more than $12,000 / \mathrm{mm}^{3}$ or leucopenia (total leucocyte count less than $4,000 / \mathrm{mm}^{3}$ ), tachycardia (heart rate more than 90 beats/min) or tachypnea (respiratory rate more than 20 beats/min). Sepsis is defined as life-threatening organ dysfunction caused by a dysregulated host response to infection. ${ }^{14}$ Sepsis secondary to urinary tract infection was considered as urosepsis. 
All the pre, intra and post-operative data were collected in a preformed proforma and entered into Microsoft Excel spreadsheet 2013. The data were checked for consistency and validity. They were then imported to Statistical Package for Social Sciences (SPSS ${ }^{\mathrm{TM}}$ ) software version 16 for statistical analysis. Categorical variables were analysed as the Fisher exact test and chi-square tests to determine associations among the various groups and subgroups. Sensitivity, specificity, Positive Predictive Value (PPV), Negative Predictive Value (NPV) and association risks were calculated. $\mathrm{P}$ value less than 0.05 was considered statistically significant.

\section{RESULTS}

There were 140 patients which included 73 males and 67 females. The mean age of the patient was $39.9 \pm 14.8$ years. The mean operation time was $40.61 \pm 13.4$ minutes. Table 1 shows mean age, mean operation time and stone burden of the patients under study.

Table 1: Patient and stone demographics

\begin{tabular}{lc}
\hline Mean Age \pm SD(in years) & $39.97 \pm 14.87$ \\
Mean Stone Burden \pm SD $\left(\right.$ in $\left.\mathrm{mm}^{2}\right)$ & $190.97 \pm 177.5$ \\
Mean Operative Time \pm SD ((in minute) & $40.61 \pm 13.4$ \\
\hline
\end{tabular}

PMUC was the most prevalent culture positive specimen with 21 cases (15\%) with Escherichia coli (7.1\%) being the commonest organism Isolated. RPUC was positive in 11 cases $(7.9 \%)$ with Proteus mirabilis (3.6\%) infection being the commonest organism cultured. Stone culture was positive in six cases (4.3\%) where Proteus mirabilis $(1.4 \%)$ was the commonest organism cultured. Table 2 depicts the same.

Table 2: Types of organism obtained in PMUC, RPUC and RSC

\begin{tabular}{lcc}
\hline PMUC positive Organisms cultured $(\mathbf{N}=\mathbf{2 1})$ & Frequency & Percent \\
Staphylococcus aureus & 1 & 0.7 \\
Mixed growth & 1 & 0.7 \\
Klebsiella & 4 & 2.9 \\
Proteus mirabilis & 5 & 3.6 \\
E.coli & 10 & 7.1 \\
RPUC positive Organisms cultured $(\mathrm{N}=11)$ & Frequency & Percent \\
E.coli & 3 & 2.1 \\
Klebsiella & 3 & 2.1 \\
Proteus mirabilis & 5 & 3.6 \\
RSC positive Organisms cultured $(\mathrm{N}=6)$ & Frequency & Percent \\
E.coli & 1 & 0.7 \\
Klebsiella & 1 & 0.7 \\
Staphylococcus aureus & 1 & 0.7 \\
Pseudomonas & 1 & 0.7 \\
Proteus mirabilis & 2 & 1.4 \\
\hline
\end{tabular}

None of the patients had simultaneous culture positivity in all the three types of specimens. Only two (1.42\%) patients had simultaneous positivity in pelvic urine culture and stone culture. The organisms obtained in pelvic urine culture and stone cultures were same i.e. Klebsiella and E.coli respectively. Only two (1.42\%) patients developed SIRS post $P C N L$, where in both the cases stone culture were positive but PMUC and RPUC were negative. Urosepsis was found in none of the patients.

Detection of infection of preoperative midstream urine culture and renal pelvic urine culture were correlated with renal stone culture for the detection of infection in renal stones. True and false positivity along with true and false negativity were calculated for the same, which is depicted in the table no. 3

Table 3: Table showing parameters of PMUC and RPUC for the detection of infection in renal stones.

\begin{tabular}{lcc}
\hline Parameters & Preoperative midstream urine c/s & Renal pelvic urine c/s \\
True Positive & 1 & 2 \\
True Negative & 114 & 125 \\
False Positive & 20 & 9 \\
False Negative & 5 & 4 \\
\hline
\end{tabular}

PMUC and RPUC didn't show significant association with RSC on Fisher exact test where p value was 1 and 0.071 . PMUC in our study showed $16.67 \%$ sensitivity, $85.07 \%$ specificity, positive predictive value of $4.76 \%$, and negative predictive value of $95.8 \%$ in $95 \%$ confidence interval (CI), in the detection of infection in renal stones. Likewise, renal pelvic urine showed $33.33 \%$ sensitivity, 93.28\% specificity, $18.18 \%$ of positive predictive value, $96.9 \%$ of negative predictive value in $95 \% \mathrm{CI}$ in the detection of infection in renal stones which is shown in table 4.

Table 4: Predicting infected stones with preoperative midstream urine and renal pelvic urine

\begin{tabular}{lcc}
\hline \multicolumn{1}{c}{$\begin{array}{c}\text { Diagnostic } \\
\text { accuracy }\end{array}$} & $\begin{array}{c}\text { Preoperative midstream urine } \\
\text { c/s (\%) }\end{array}$ & $\begin{array}{c}\text { Renal pelvic urine } \\
\mathbf{c} / \mathbf{s}(\%)\end{array}$ \\
Sensitivity & 16.67 & 33.33 \\
Specificity & 85.07 & 93.28 \\
$\begin{array}{l}\text { Positive predictive } \\
\text { value (PPV) }\end{array}$ & 4.76 & 18.18 \\
$\begin{array}{l}\text { Negative predic- } \\
\text { tive value (NPV) }\end{array}$ & 95.80 & 96.90 \\
\hline
\end{tabular}

PMUC had $0 \%$ sensitivity, $84.7 \%$ specificity, $0 \%$ positive predictive value and $98.3 \%$ of negative predictive value in $95 \% \mathrm{CI}$ for the detection of SIRS in post PCNL patient, where as it was $0 \%$ sensitivity, $92.03 \%$ specificity, $0 \%$ of positive predictive value and $98.45 \%$ of negative predictive value in $95 \% \mathrm{CI}$ in RPUC for the detection of SIRS post PCNL. RSC on the other hand had sensitivity of $100 \%$, specificity of $97.10 \%$, and positive predictive value of 
$33.33 \%$ and negative predictive value of $100 \%$ with $95 \%$ of $\mathrm{CI}$ in the detection of SIRS in post PCNL patients. In the Fischer Exact test PMUC and RPUC were not statistically significant in the detection of SIRS post PCNL the $p$ value was 1 in both the cases respectively. Whereas RSC showed statistical significance in the detection of SIRS in the Fischer Exact test $\mathrm{p}$ value is 0.002 . Table 5 depicts the same.

Table 5: Predicting SIRS using various specimens

\begin{tabular}{lccc} 
Diagnostic accuracy & PMUC (\%) & RPUC (\%) & RSC (\%) \\
Sensitivity & 0.00 & 0.00 & 100 \\
Specificity & 84.78 & 92.03 & 97.10 \\
PPV & 0 & 0 & 33.33 \\
NPV & 98.32 & 98.45 & 100 \\
P value $(95 \% \mathrm{Cl})$ & 1 & 1 & 0.002 \\
\hline
\end{tabular}

\section{DISCUSSION}

Postoperative fever in PCNL is bothersome which ranges from $10-15 \% \cdot{ }^{15,16}$ Fever post PCNL can be due to underlying infection, hence we routinely gave antibiotic prophylaxis three days prior to surgery according to our local hospital protocol. And in cases where pre-operative midstream urine culture was positive, antibiotics were given unless the urine culture report came out to be negative.

Positive preoperative midstream urine culture in our study was $15 \%$ and E.coli was the commonest organism detected which is comparable to the study conducted by Gutierrez et al. ${ }^{1}$ But in a study conducted by Patel et $\mathrm{al}^{17}$, PMUC was $51 \%$ which is much higher than our study and the reason for it might be due to the absence of patients in our study who were under long-term catheters, nephrostomy tubes. ${ }^{1}$ We also aimed to make preoperative urine sterile even in stag horn stones.

Preoperative midstream urine culture is not a good test for the presumption of SIRS or urosepsis post PCNL which is shown by Mariappan et $\mathrm{al}^{18}$ and Paonessa et al. ${ }^{19}$ Sensitivity of preoperative midstream urine culture and the pelvic urine culture for the detection of infected stones were $16.67 \%$ and $33.33 \%$ respectively in our study which is comparable to the study conducted by Mariappan et al. ${ }^{18}$ Likewise, stone culture has the highest value in detecting SIRS/urosepsis as according to the above mentioned studies, which is comparable to the findings of our study.

SIRS in our patient was only $1.42 \%$ and no urosepsis was noted, which is much less compared to the study done by Waltondiaz et al. ${ }^{11}$ The reason might be due to stringent use of antibiotics preoperatively and continuation of the same postoperatively for seven days in our study.
The present study has few limitations as the surgeries were performed by different consultants. Robust methods for the calculation of stone burden was not carried out. It was a single center study.

\section{CONCLUSION}

Stone culture has high prediction for SIRS and it should be considered for patients undergoing PCNL in order to prevent stone related infective complications.

\section{Conflict of interest}

Authors declare no conflict of interest

\section{REFERENCES}

1. Gutierrez J, Smith A, Geavlete P, Shah H, Kural AR, de Sio M, et al. Urinary tract infections and post-operative fever in percutaneous nephrolithotomy. World J Urol. 2013 Oct;31(5):1135-40. PMID: 22367718.

2. Alyami FA, Skinner TAA, Norman RW. Impact of body mass index on clinical outcomes associated with percutaneous nephrolithotomy. Can Urol Assoc J. 2013;7(3-4):197-201. PMID: 22630337.

3. Türk C, Petř́́k A, Sarica K, Seitz C, Skolarikos A, Straub $\mathrm{M}$, et al. EAU Guidelines on Interventional Treatment for Urolithiasis. Eur Urol. 2016 Mar;69(3):475-82. PMID: 26344917.

4. Shin TS, Cho HJ, Hong S-H, Lee JY, Kim SW, Hwang TK. Complications of Percutaneous Nephrolithotomy Classified by the Modified Clavien Grading System: A Single Center's Experience over 16 Years. Korean J Urol. 2011 Nov 1;52(11):769-75. PMID: 22195267.

5. Gonzalez-Ramirez A, Camarena L, Gutierrez-Aceves J. Risk factors for fever and sepsis after percutaneous nephrolithotomy. J Urol. 2013; 4 Supplement (189):e633. DOI: 10.1016/j.juro.2013.02.3034

6. Kreydin EI, Eisner BH. Risk factors for sepsis after percutaneous renal stone surgery. Nat Rev Urol. 2013 Oct;10(10):598-605. PMID: 23999583.

7. Tuzel E, Aktepe OC, Akdogan B. Prospective Comparative Study of Two Protocols of Antibiotic Prophylaxis in Percutaneous Nephrolithotomy. J Endourol. 2013Feb;27(2):172-6. PMID: 22908891.

8. Lojanapiwat B. Infective complication following percutaneous nephrolithotomy. Urol Sci. 2016 Mar 1;27(1):8-12. DOI:10.1016/j.urols.2015.04.007

9. Rashid AO, Fakhulddin SS. Risk factors for fever and sepsis after percutaneous nephrolithotomy. Asian J Urol. 2016 Apr;3(2):82-87. PMID: 29264169. 
10. Koras O, Bozkurt IH, Yonguc T, Degirmenci T, Arslan B, Gunlusoy B, et al. Risk factors for postoperative infectious complications following percutaneous nephrolithotomy: a prospective clinical study. Urolithiasis. 2015 Feb 1;43(1):55-60. PMID: 25269441.

11. Walton-Diaz A, Vinay JI, Barahona J, Daels P, González $\mathrm{M}$, Hidalgo JP, et al. Concordance of renal stone culture: PMUC, RPUC, RSC and post-PCNL sepsis-a nonrandomized prospective observation cohort study. Int Urol Nephrol. 2017 Jan;49(1):31-5. DOI: 10.1007/ s11255-016-1457-y

12. Alelign T, Petros B. Kidney Stone Disease: An Update on Current Concepts. Adv Urol. 2018 Feb 4; 2018. DOI:10.1155/2018/3068365

13. Ohio BNB PharmD, BCPSClinical Assistant Professor of Pharmacy PracticeOhio Northern UniversityAda, OhioClinical PharmacistLima Memorial HospitalLima. Interpretation of Urinalysis and Urine Culture for UTI Treatment. US Pharm. 2013;38(11):65-68.

14. Singer M, Deutschman CS, Seymour CW, Shankar-Hari M, Annane D, Bauer M, et al. The Third International Consensus Definitions for Sepsis and Septic Shock (Sepsis-3). JAMA. 2016 Feb 23;315(8):801-10. DOI: 10.1001/jama.2016.0287

15. Draga ROP, Kok ET, Sorel MR, Bosch RJLH, Lock TMTW. Percutaneous nephrolithotomy: factors associated with fever after the first postoperative day and systemic inflammatory response syndrome. J Endourol. 2009 Jun;23(6):921-7. DOI: 10.1089/ end.2009.0041

16. Deshmukh S, Sternberg K, Hernandez N, Eisner BH.
Compliance with American Urological Association Guidelines for Post-Percutaneous Nephrolithotomy Antibiotics Does Not Appear to Increase Rates of Infection. J Urol. 2015 Oct;194(4):992-6. PMID: 25936864.

17. Patel N, Shi W, Liss M, Raheem O, Wenzler D, Schallhorn C, et al. Multidrug resistant bacteriuria before percutaneous nephrolithotomy predicts for postoperative infectious complications. J Endourol. 2015 May;29(5):531-6. PMID: 25424241.

18. Mariappan P, Smith G, Bariol SV, Moussa SA, Tolley DA. Stone and pelvic urine culture and sensitivity are better than bladder urine as predictors of urosepsis following percutaneous nephrolithotomy: a prospective clinical study. J Urol. 2005 May;173(5):1610-4. PMID: 15821509.

19. Paonessa JE, Gnessin E, Bhojani N, Williams JC, Lingeman JE. Preoperative Bladder Urine Culture as a Predictor of Intraoperative Stone Culture Results: Clinical Implications and Relationship to Stone Composition. J Urol. 2016 Sep;196(3):769-74. PMID: 27038771. 